\title{
Phenomenological models of the transient processes of diesel spray tip penetration
}

\author{
Xinyi Zhou ${ }^{1,2}$, Tie Li*1,2, Ping $\mathrm{Yi}^{1,2}$, Ning Wang ${ }^{1,2}$ \\ ${ }^{1}$ State Key Laboratory of Ocean Engineering, Shanghai Jiao Tong University, Shanghai, PR \\ China \\ ${ }^{2}$ Institute of Power Plants and Automation, Shanghai Jiao Tong University, Shanghai, PR \\ China \\ *Corresponding author email : litie@sjtu.edu.cn
}

\begin{abstract}
The multiple-injection strategy that has been used widely in diesel engines usually features a short duration for each injection pulse, which makes the start-of-injection (SOI) and end-ofinjection (EOI) transients increasingly important for sprays in an injection event. Owing to the needle movement, spray developments during the transient processes are quite different from the spray at the quasi-steady state. In this paper, considering the sac pressurization processes during the SOI transients and effects of "entrainment wave" after the EOI, a theoretical zerodimensional (0-D) model for the entire development processes of spray tip penetration is deduced. Then, the model is validated against the experimental spray data using a constant volume chamber and high-speed shadowgraphy. The model and experimental results demonstrate that the spray tip penetration has a $t^{3 / 2}$ dependence at the initial stage of injection rather than the $t$ dependence suggested by the Hiroyasu model. Later, the spray tip penetration has a $t^{3 / 4}$ dependence owing to the spray breakup, a $t^{1 / 2}$ dependence with the completion of sac pressurization, and a $\left(t-t_{i}\right)^{1 / 4}$ dependence after two injection durations from the SOI ${ }^{1}$.
\end{abstract}

\section{Keywords}

Diesel spray; Start-of-injection; End-of-injection; Spray tip penetration; 0-D models

\section{Introduction}

Spray tip penetration $\left(S_{t i p}\right)$ is one of the most important spray characteristics for diesel engines. As a result, accurate and quick calculation of $S_{\text {tip }}$ is important for optimization of the spray combustion system. Because of its simplicity and ability to clearly reflect the effects of various design parameters, the 0-D model has been widely used in model-based optimization of diesel spray combustion systems.

With the assumption that the diesel spray is approximated as a gas jet, Wakuri et al. [1] developed a model for $S_{t i p}$ based on the theory of momentum conservation. They found that $S_{t i p}$ is proportion to the square root of time $t^{0.5}$. The $t^{0.5}$ dependence was verified by many studies [2]. Hiroyasu et al. [3] demonstrated that $S_{\text {tip }}$ is initially proportional to the time $t$ and then proportional to $t^{0.5}$. They derived a correlation based on Levich's jet disintegration theory [4]. Recently, the model constants of Hiroyasu's model were modified by Arai [5] for higher fuel injection pressures. Naber and Siebers [6] modified the Wakuri model [1] by considering a turbulent two-phase jet. According to their theoretical derivation, the dependence of $S_{\text {tip }}$ upon the time gradually transitions from $t$ to $t^{0.5}$, which confirms the correctness of Hiroyasu's model [3].

\footnotetext{
${ }^{1} t$ denotes the time after start of the fuel injection, and $t_{i}$ the injection duration.
} 
In the derivation processes of the above models, an idealized rectangular fuel injection rate profile was assumed [1-6], and the increase of sac pressure during the start-of-injection (SOI) transients and the termination of fuel momentum supply after the end-of-injection (EOI) were not considered. Recently, the above models were found to give an unacceptable prediction of $S_{\text {tip }}$ during the SOI transients and after the EOI $[7,8]$. These limitations seem to be acceptable when using a single long-pulsed fuel injection [1-6], where the SOI transients account for a relatively small proportion of the entire injection processes and the spray usually impinges upon the wall before the EOI. For modern diesel engines employing multiple-injection strategies, the SOI transients play an important role in the entire injection processes and spray evolution after the EOI can not be ignored, especially for the low temperature combustion that features in the early fuel injection. Therefore, further studies about $S_{\text {tip }}$ during the SOI transients and after the EOI are needed.

Kostas et al. [9] studied $S_{\text {tip }}$ during the initial $0.5 \mathrm{~ms}$ after SOI, and they found that $S_{\text {tip }}$ follows an empirical correlation $S_{t i p}(t)=A t^{1.5}$ during the SOI transients. They suggested using their correlation in conjunction with Hiroyasu's correlation to describe the whole $S_{t i p}$ behaviour. Following the $t^{1.5}$ dependence suggested by Kostas et al. [9], Taşkiran et al. [10] modified the empirical correlation for the initial $S_{t i p}$ by considering the effects of injection pressure and ambient density. They confirmed that $S_{t i p}$ has a $t^{1.5}$ dependence at the injection startup. Recently, the authors of this paper proposed a theoretical model for $S_{\text {tip }}$ during the SOI transients [8]. They reported that $S_{\text {tip }}$ is proportional to $t^{1.5}$ at the acceleration stage, $t^{0.75}$ or $t^{1}$ at the transition stage, and $t^{0.5}$ at the quasi-steady stage. They found that the model prediction results are in great agreement with the experimental data.

There are also many studies on spray evolution after the EOI. For example, Musculus et al. [11] developed a 1-D model for $S_{\text {tip }}$ during the EOI transients. They reported that $S_{\text {tip }}$ gradually transitions to be proportional to $t^{0.25}$ after two injection durations. Liu et al. [12] developed a 0 $\mathrm{D}$ model for $S_{\text {tip }}$ during the EOI transients. They confirmed that $S_{t i p}$ is proportional to $t^{0.25}$ when the time is much longer than the injection duration. The time dependence $t^{0.25}$ has also been observed for a small quantity diesel spray [13] and water-jet [14]. The authors of this paper modified the fuel injection speed in Liu's model to the fuel injection pressure and made the changed model continuous with the Hiroyasu model at the time of two injection durations, and proposed a model for spray tail penetration based on the discrete control volume method [7]. Recently, Zhou et al. [15] studied the spray evolution during the EOI transient and compared various models developed so far. They reported that only the present authors' model [7] exhibits a trend close to the experimental results.

As reviewed in the above paragraph, although $S_{\text {tip }}$ during the SOI and EOI transients have been studied to some extent, so far no models are summarized to predict the entire development processes of $S_{\text {tip }}$. More unfortunately, there are almost no data that can be used to develop and validate such a model. This is because the existing studies about the SOI and EOI transients are independent of each other. One of the objectives of this paper is to summarize the present authors' previous work on the transient diesel spray [7, 8] and further develop a model for the entire development processes of $S_{t i p}$. Another objective is to provide experimental data to verify the newly developed model.

\section{Model formulation}

Figure 1(a) shows the conceptual models for the entire development process of $S_{\text {tip }}$ when $t_{p}$ is smaller than $t_{b}$. As can be seen, there are four key time points in the entire development process of $S_{t i p}$ : the sac pressurization time $\left(t_{p}\right)$, breakup time $\left(t_{b}\right)$, injection duration $\left(t_{i}\right)$ and two injection durations $\left(2 t_{i}\right)$. Five stages can be divided according to these four time points: the 
acceleration stage, transition stage 1, quasi-steady stage, transition stage 2 and decelerating stage.

(a) Sac pressurization time $\left(t_{p}\right)<$ Breakup time $\left(t_{b}\right)$

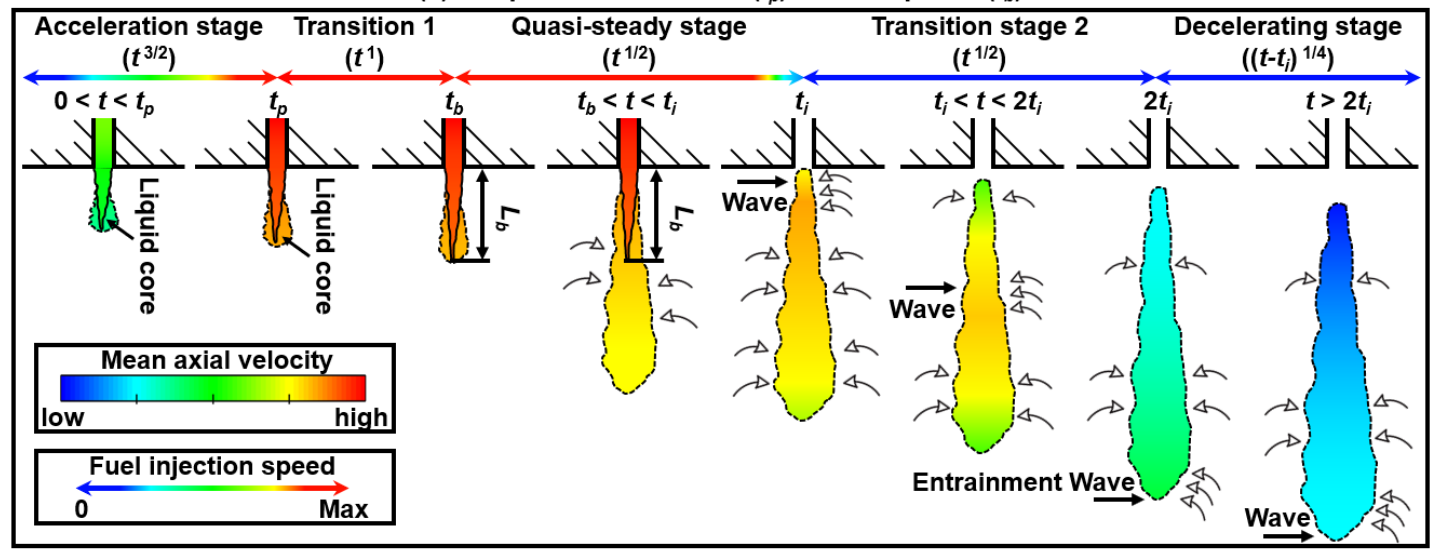

(b) Sac pressurization time $\left(t_{p}\right)>$ Breakup time $\left(t_{b}\right)$

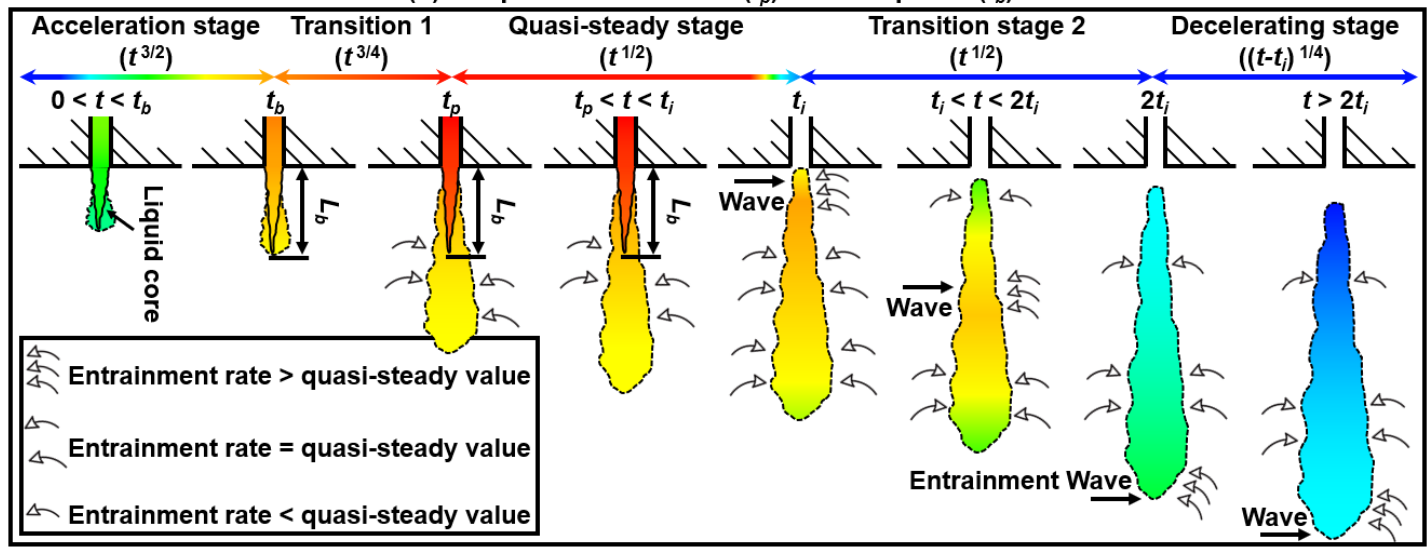

Figure 1. Conceptual models of the entire development processes of $S_{t i p}$.

(1) At the acceleration stage $\left(0<t \leq t_{p}\right)$, the sac pressurization processes have not been completed and the liquid core is intact from the nozzle exit to the spray tip. During this time, the nozzle exit fuel velocity gradually increases and the increased fuel momentum at the nozzle exit would be easily transferred to the spray tip. As a result, $S_{t i p}$ exhibits an acceleration behavior during this stage.

(2) At the transition stage $1\left(t_{p} \leq t \leq t_{b}\right)$, the sac pressurization processes has been completed but $S_{t i p}$ is still smaller than the breakup length. At this point, $S_{t i p}$ exhibits a linear dependence upon time, which is consistent with the Hiroyasu model before spray breakup.

(3) At the quasi-steady stage $\left(t_{b} \leq t \leq t_{i}\right)$, since the breakup length is obviously shorter than the spray penetration length, $S_{\text {tip }}$ during this stage is mainly controlled by fuel-air mixing. As a result, the late-injected fuel finds it is difficult to catch up to the early-injected fuel and $S_{\text {tip }}$ exhibits a deceleration behavior.

(4) At the transition stage $2\left(t_{i} \leq t \leq 2 t_{i}\right)$, a disturbance of increased air entrainment termed the "entrainment wave" gradually travels downstream from the nozzle outlet to the spray tip. Since the "entrainment wave" has not arrived, the spray tip still remains at the quasi-steady stage before $2 t_{i}$ and the same model in the quasi-steady stage can be used.

(5) At the decelerating stage $\left(2 t_{i} \leq t\right)$, the whole spray enters into the decelerating state and the total momentum at the spray tip decreases with the time elapsing. As a result, the velocity of the spray tip is further reduced during this stage.

Figure 1(b) shows the conceptual models of the entire development processes of $S_{t i p}$ when $t_{p}$ 
is larger than $t_{b}$. $S_{t i p}$ behavior at the acceleration stage, quasi-steady stage, transition stage 2 and decelerating stage is consistent with the situation when $t_{p}$ is smaller than $t_{b}$. At the transition stage $1\left(t_{b} \leq t \leq t_{p}\right)$, the sac pressurization processes are still incomplete but $S_{t i p}$ is longer than the breakup length. The fuel moving inside the liquid core penetrates to the breakup length with negligibly small velocity loss, then the fuel moves to the spray tip with a gradually decreased velocity after breakup owing to the momentum exchange between the ambient air and fuel. As a result, although the nozzle exit fuel velocity still increases during this stage, $S_{t i p}$ exhibits a slight deceleration behavior.

As discussed in the above paragraphs, the Hiroyasu model [3] can be used at the quasi-steady stage and the transition stage 2 . Based on the relationship among sac pressure, needle lift and time after start of injection (aSOI), the authors of this paper proposed a theoretical model for $S_{t i p}$ at the acceleration stage and the transition stage 1 [8]. Moreover, a theoretical model for $S_{t i p}$ at the decelerating stage is developed by the present authors [7]. Making the models in $[3,7-8]$ continuous at the turning points leads to

$$
\begin{aligned}
& \begin{cases}0<t \leq t_{p} & S_{t i p}(t)=K_{1}\left(2 \Delta P / t_{p}\right)^{1 / 2} \rho_{f}^{-1 / 2} t^{3 / 2} \\
t_{p} \leq t \leq t_{b} & S_{t i p}(t)=K_{1}\left(2 \Delta P / \rho_{f}\right)^{1 / 2} t \\
t_{b} \leq t \leq 2 t_{i} & S_{t i p}(t)=K_{2}\left(\Delta P / \rho_{a}\right)^{1 / 4}\left(d_{n}\right)^{1 / 2}(t)^{1 / 2} \\
2 t_{i} \leq t & S_{t i p}(t)=2^{1 / 2} K_{2}\left(\Delta P / \rho_{a}\right)^{1 / 4}\left(d_{n}\right)^{1 / 2} t_{i}^{1 / 4}\left(t-t_{i}\right)^{1 / 4} \\
t_{b}=K_{b t}\left(\rho_{f} d_{n}\right)\left(\rho_{a} \Delta P\right)^{-1 / 2} \quad K_{2}=K_{1}\left(2 K_{b t}\right)^{1 / 2}\end{cases} \\
& \begin{cases}0<t \leq t_{b} & S_{t i p}(t)=K_{1}\left(2 \Delta P / t_{p}\right)^{1 / 2} \rho_{f}^{-1 / 2} t^{3 / 2} \\
t_{b} \leq t \leq t_{p} & S_{t i p}(t)=K_{2}\left(\Delta P / t_{p}\right)^{1 / 4} \rho_{a}^{-1 / 4}\left(d_{n}\right)^{1 / 2}(t)^{3 / 4} \\
t_{b} \leq t \leq 2 t_{i} & S_{t i p}(t)=K_{2}\left(\Delta P / \rho_{a}\right)^{1 / 4}\left(d_{n}\right)^{1 / 2}(t)^{1 / 2} \\
2 t_{i} \leq t & S_{t i p}(t)=2^{1 / 2} K_{2}\left(\Delta P / \rho_{a}\right)^{1 / 4}\left(d_{n}\right)^{1 / 2} t_{i}^{1 / 4}\left(t-t_{i}\right)^{1 / 4} \\
t_{b}=K_{b t}\left(\rho_{f} d_{n}\right)\left(\rho_{a} \Delta P\right)^{-1 / 2} \\
K_{2}=2^{1 / 2} K_{1} K_{b t}^{3 / 4} \Delta P^{-1 / 8} t_{p}^{-1 / 4} \rho_{f}^{1 / 4} d_{n}^{1 / 4} \rho_{a}^{-1 / 8}\end{cases}
\end{aligned}
$$

here, $t$ is the time after the start of injection [s]; $\rho_{a}$ refers to the ambient density $\left[\mathrm{kg} / \mathrm{m}^{3}\right] ; d_{n}$ is the nozzle hole diameter [m]; $\triangle P$ indicates the difference between fuel injection pressure and ambient pressure $[\mathrm{Pa}] ; \rho_{f}$ is the fuel density $\left[\mathrm{kg} / \mathrm{m}^{3}\right] ; K_{1}$ and $K_{2}$ are the model constants; $K_{b t}$ is the breakup time factor. Equation (1) is suitable when $t_{p}$ is smaller than $t_{b}$, while Equation (2) is suitable when $t_{p}$ is larger than $t_{b}$. It can be seen that both sides of Equations (1) and (2) can satisfy the conservation of dimensions, which verifies the correctness of the models.

According to Equations (1) and (2), $S_{\text {tip }}$ shows a $t^{1.5}$ dependence upon time at the acceleration stage, $t^{1}$ or $t^{0.75}$ dependence at the transition stage $1, t^{0.5}$ dependence at the quasi-steady stage and transition stage 2 , and $\left(t-t_{i}\right)^{0.25}$ dependence at the decelerating stage. Since the exponent gradually decreases from 1.5 to 0.25 , the spray tip experiences an acceleration process at the injection startup and then moves downstream at a gradually reduced speed.

\section{Experimental setups}

The Bosch long-tube method was used in the present study to measure the fuel injection rate, more details can be found in [16]. A constant volume combustion chamber (CVCC) was employed to measure the spray characteristics. The transient pressure in the CVCC was obtained by a pressure transducer (Kistler 6054B). One injector adapter was mounted on the head of the chamber, while five optical windows of $100 \mathrm{~mm}$ diameter can be mounted on the 
bottom and sides of the chamber. Moreover, the distance from the nozzle outlet to the field of view was changed by using different injector adapters, as shown in Figure 2. In this study, the injector adapter 1 in Figure 2(a) was used to obtain $S_{\text {tip }}$ between 0 to $90 \mathrm{~mm}$ (i.e. the spray upstream), while the injector adapter 2 in Figure 2(b) was used to obtain $S_{\text {tip }}$ between 90 to $180 \mathrm{~mm}$ (i.e. the spray downstream). Figure 2(c) illustrates the diffused back-illumination (DBI) setup for imaging the temporal development of spray. An in-house light emission diode (LED) was adopted as the light source. After passing through a diffuser and the spray region, the light was captured by a CMOS camera coupled with a Nikon lens. One can refer to previous studies for more information about the optical arrangements [17]. Details about the imaging parameter settings and the experimental conditions are shown in Table 1.

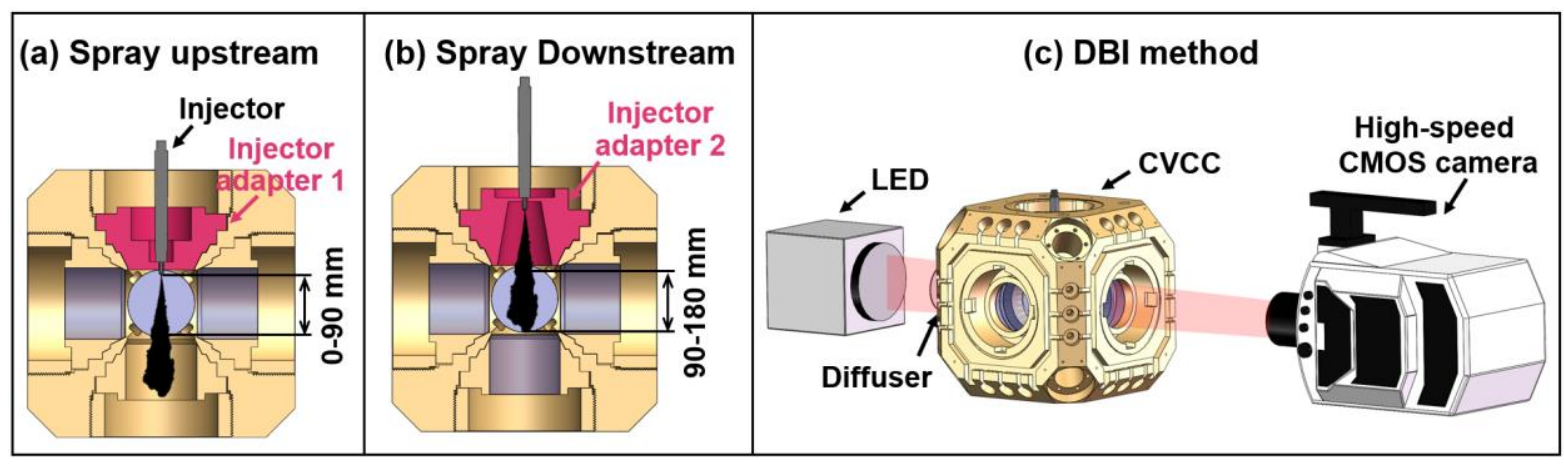

Figure 2. Experimental setups: (a) injector adapter 1 for the spray upstream; (b) injector adapter 2 for the spray downstream; (c) high-speed diffused back illumination imaging.

Table 1 - Experimental conditions and imaging settings.

\begin{tabular}{l|l}
\hline Parameter & Value \\
\hline Nozzle hole diameter $(\mathrm{mm})$ & 0.28 \\
Fuel injection pressure $(\mathrm{MPa})$ & 120 \\
Injection duration $(\mathrm{ms})$ & 2 \\
Ambient density $\left(\mathrm{kg} / \mathrm{m}^{3}\right)$ & $20,40,60$ \\
Ambient temperature $(\mathrm{K})$ & 293 \\
Ambient gas & Nitrogen \\
Imaging speed $(\mathrm{fps})$ & 80,000 \\
Exposure time $(\mu \mathrm{s})$ & 1 \\
\hline
\end{tabular}

\section{Experimental results}

Figure 3 shows the fuel injection rate profile with $120 \mathrm{MPa}$ fuel injection pressure and $2 \mathrm{~ms}$ injection duration. According to the methods proposed in $[8,18]$, the sac pressurization time can be approximated as the time of the rapid increase of the injection rate at the injection startup. In this study, the sac pressurization time is determined to be $0.34 \mathrm{~ms}$ based on the ramp-up processes in Figure 3. Figure 4(a) shows the evolutions of $S_{\text {tip }}$ under different ambient densities. The solid, half hollow and hollow symbols are used to distinguish the data from SOI to $t_{i}$, from $t_{i}$ to $2 t_{i}$, and after $2 t_{i}$, respectively. Since the momentum exchange between the ambient air and fuel is enhanced at the higher ambient density, the $S_{\text {tip }}$ decreases with the ambient density increasing. The spray tip velocity $\left(V_{t i p}\right)$ can be calculated by the derivative of $S_{t i p}$, as given in Figure 4(b). It can be seen that $V_{\text {tip }}$ increases rapidly during the initial stage of injection and then gradually decreases to a lower value, which is consistent with the analyses of Figure 1(b). During the acceleration stage, the liquid core is relatively intact, and $V_{\text {tip }}$ mainly depends on the difference between fuel injection pressure and ambient pressure. 
Since the ambient pressure is negligibly small compared to the fuel injection pressure, the effects of ambient density on $V_{\text {tip }}$ is relatively small during the acceleration stage. After the acceleration stage, when $S_{t i p}$ is longer than the breakup length, the ambient air entrainment rate plays an important role in affecting $V_{\text {tip }}$. As a result, $V_{t i p}$ decreases with ambient density increasing after the acceleration stage. In the next section, the data in Figure 4(a) will be used to verify the newly developed model of this paper.

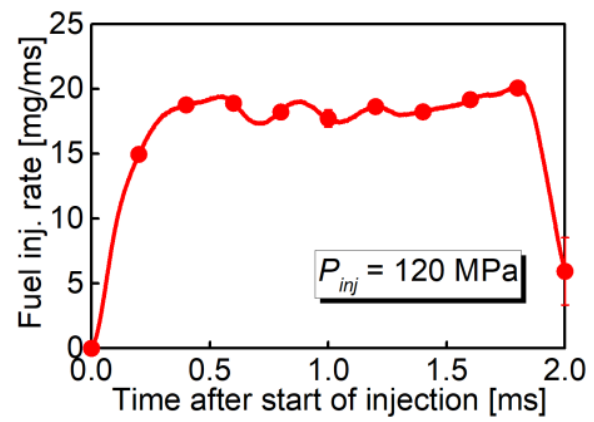

Figure 3. Fuel injection rate profile with $120 \mathrm{MPa}$ fuel injection pressure and 2 ms injection duration

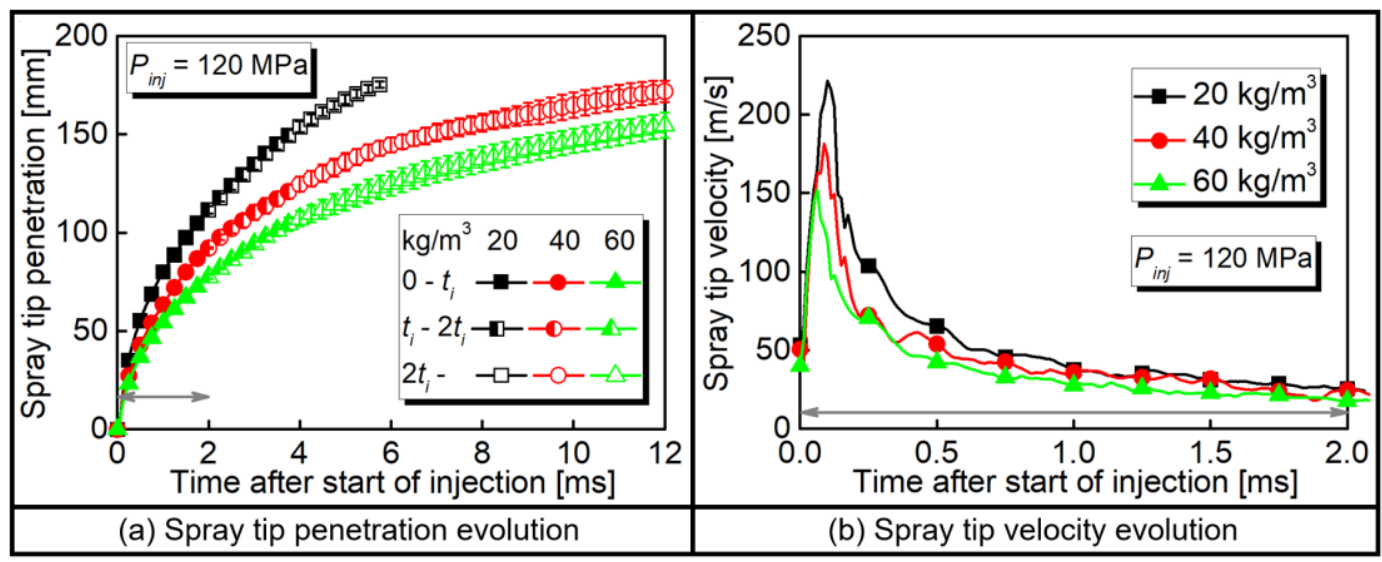

Figure 4. Spray tip penetration and spray tip velocity evolutions under different ambient densities.

\section{Model evaluation}

For the experimental conditions of this study, the sac pressurization time is larger than the breakup time. As a result, Equation (2) is applicable. Owing to the relationship between $K_{1}$ and $K_{2}$, there is only one model constant in Equation (2) that should be calibrated to consider the effects of different nozzle geometries. Calibrating $K_{1}$ against the experimental $S_{\text {tip }}$ data, the optimal $K_{1}$ is 0.51 with the determination coefficient $R^{2}$ up to $98.7 \%$, as shown in Figure 5. It can be seen that the calculated results agree very well with the experimental data.

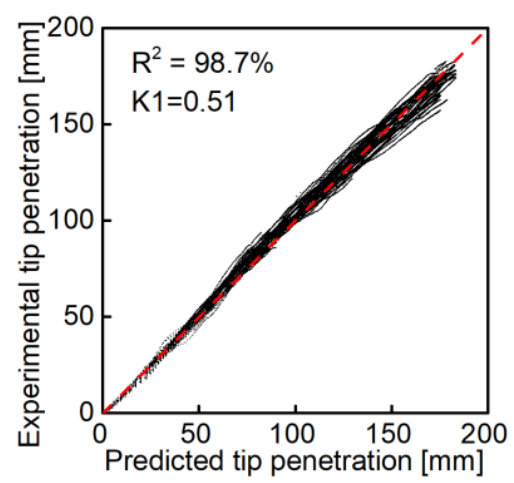

Figure 5. Comparison of $S_{\text {tip }}$ between the experiment and calculation. 
Figure 6 shows a comparison of the newly developed model and the Hiroyasu model under different ambient densities. The left side of Figure 6 uses the linear coordinates to observe the overall evolution, while the right side uses logarithmic coordinates to better compare the predictability of these two models at the injection startup. The black, dark gray and light gray dots represent the experimental data from SOI to $t_{i}$, from $t_{i}$ to $2 t_{i}$ (i.e. the transition stage 2) and after $2 t_{i}$ (i.e. the decelerating stage), respectively. Since the sac pressurization processes at the injection startup are not considered in the derivation processes, the Hiroyasu model overpredicts $S_{t i p}$ at the initial stage of injection. Moreover, the Hiroyasu model significantly overpredicts $S_{\text {tip }}$ after $2 t$, this is because that the quasi-steady fuel injection is assumed and the termination of fuel injection and fuel momentum supply is not considered in its derivation processes. Since the sac pressurization processes during the SOI transients and effects of "entrainment wave" after the EOI are considered, the newly developed model can predict the entire development processes of $S_{t i p}$. It should be noted that $K_{b t}$ of 28.65 suggested by Hiroyasu et al [3] is used for the newly developed model. As a result, in the logarithmic coordinates, the time of the first turning point of the newly developed model and the Hiroyasu model is consistent.

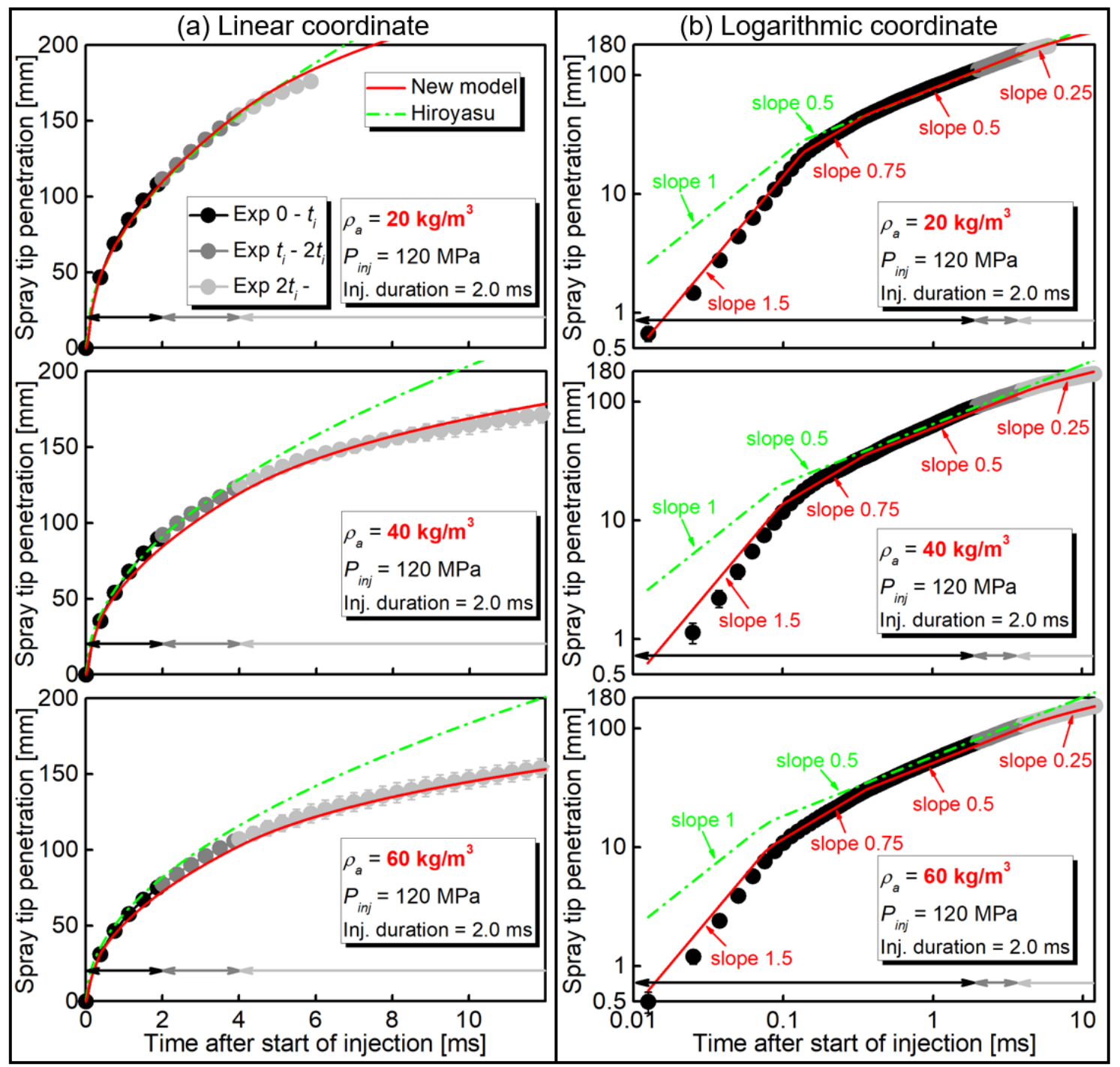

Figure 6. Model evaluation under the different ambient densities: (a) using the linear coordinate; (b) using the logarithmic coordinate. 


\section{Conclusions}

The model and experimental results clarify four key time points in the entire development processes of $S_{t i p}$ : the sac pressurization time $\left(t_{p}\right)$, breakup time $\left(t_{b}\right)$, injection duration $\left(t_{i}\right)$ and two injection durations $\left(2 t_{i}\right)$.

The entire development processes of $S_{\text {tip }}$ can be summarized into five stages: the acceleration stage $\left(S_{\text {tip }}\right.$ proportion to $\left.t^{1.5}\right)$, transition stage $1\left(t^{1}\right.$ or $\left.t^{0.75}\right)$, quasi-steady stage $(t$ $0.5)$, transition stage $2\left(t^{0.5}\right)$ and decelerating stage $\left(\left(t-t_{i}\right)^{0.25}\right)$.

The calculated results of the newly developed model agree very well with the experimental data.

\section{Acknowledgments}

The supports by the Major International (Regional) Joint Research Project of National Natural Science Foundation of China (52020105009) are gratefully acknowledged.

\section{Nomenclature}

$\begin{array}{llll}t & \text { time after the start of injection [s] } & t_{p} & \text { sac pressurization time [s] } \\ t_{b} & \text { breakup time [s] } & t_{i} & \text { injection duration [s] } \\ \rho_{a} & \text { ambient density }\left[\mathrm{kg} / \mathrm{m}^{3}\right] & d_{n} & \text { nozzle hole diameter [m] } \\ \rho_{f} & \text { fuel density }\left[\mathrm{kg} / \mathrm{m}^{3}\right] & K_{b t} & \text { breakup time factor } \\ K_{1} & \text { model constant } & K_{2} & \text { model constant } \\ \mathrm{SOI} & \text { start-of-injection } & \mathrm{EOI} & \text { end-of-injection } \\ \triangle P & \text { difference between fuel injection pressure and ambient pressure [Pa] }\end{array}$

\section{References}

[1] Wakuri, Y., Fujii, M., Amitani, T., Tsuneya, R., 1960, Bull JSME, 3 (9), pp. 123-130.

[2] Desantes, JM., Payri, R., Salvador, FJ., Gil, A., 2006, Fuel, 85, pp. 910-917.

[3] Hiroyasu, H., and Arai, M., 1990, SAE paper 900475.

[4] Levich, VG., 1962, "Physicochemical Hydrodynamics."

[5] Arai, M., Aug. 23.-27. 2015, 13th International Conference on Liquid Atomization and Spray Systems.

[6] Naber, JD., and Siebers, DL., 1996, SAE paper 960034.

[7] Zhou, X., Li, T., Lai, Z., Wei, Y., 2019, Fuel, 237, pp. 442-456.

[8] Zhou, X., Li, T., Yi, P., 2020, International Journal of Engine Research, DOI: $10.1177 / 1468087420957852$.

[9] Kostas, J., Honnery, D., Soria, J., 2009, Fuel, 88, pp. 2225-2237.

[10] Taşkiran, Ö. O., and Ergeneman, M., 2011, Journal of Combustion, 2011, 528126.

[11] Musculus, MPB., and Kattke, K., 2009, SAE paper 2009-01-1355.

[12] Liu, L., Ma, X., Magagnato, FA., 2017, Fuel, 199, pp. 324-331.

[13] Bao, Z., Horibe, N., Ishiyama, T., 2018, SAE paper 2018-01-0283.

[14] Sangras, R., Kwon, OC., Faeth, GM., 2002, Journal of Heat Transfer, 124 (3), pp.460469.

[15] Zhou, Y., Qi, W., Zhang, Y., 2020, Energy, 211, 118605.

[16] Zhou, X., Li, T., Lai, Z., Wang, B., 2018, Fuel, 225, pp. 358-369.

[17] Zhou, X., Li, T., Wei, Y., Wang, N., 2020, International Journal of Engine Research, 21 (9), pp. 1662-1677.

[18] Zhou, X., Li, T., Yi, P., Zhang, Z., Wang, N., Wei, Y., 2020, Fuel, 276, 118026. 